\title{
Far-field Diffraction Properties of Annular Walsh Filters
}

\author{
Pubali Mukherjee $^{1}$ and Lakshminarayan Hazra ${ }^{2}$ \\ ${ }^{1}$ MCKV Institute of Engineering, 243 G.T. Road, Liluah, Howrah 711204, India \\ ${ }^{2}$ Department of Applied Optics and Photonics, University of Calcutta, 92 A.P.C. Road, Kolkata 700 009, India
}

Correspondence should be addressed to Lakshminarayan Hazra; lnhaphy@caluniv.ac.in

Received 22 March 2013; Revised 3 June 2013; Accepted 2 July 2013

Academic Editor: Augusto Belendez

Copyright (C) 2013 P. Mukherjee and L. Hazra. This is an open access article distributed under the Creative Commons Attribution License, which permits unrestricted use, distribution, and reproduction in any medium, provided the original work is properly cited.

\begin{abstract}
Annular Walsh filters are derived from the rotationally symmetric annular Walsh functions which form a complete set of orthogonal functions that take on values either +1 or -1 over the domain specified by the inner and outer radii of the annulus. The value of any annular Walsh function is taken as zero from the centre of the circular aperture to the inner radius of the annulus. The three values $0,+1$, and -1 in an annular Walsh function can be realized in a corresponding annular Walsh filter by using transmission values of zero amplitude (i.e., an obscuration), unity amplitude and zero phase, and unity amplitude and $\pi$ phase, respectively. Not only the order of the Walsh filter but also the size of the inner radius of the annulus provides an additional degree of freedom in tailoring of point spread function by using these filters for pupil plane filtering in imaging systems. In this report, we present the far-field amplitude characteristics of some of these filters to underscore their potential for effective use in several demanding applications like high-resolution microscopy, optical data storage, microlithography, optical encryption, and optical micromanipulation.
\end{abstract}

\section{Introduction}

Annular apertures and different types of ring-shaped apertures continue to be investigated for catering to the growing exigencies in diverse applications, for example, high resolution microscopy, optical data storage, microlithography, optical encryption, and optical micromanipulation [1-5]. Not only for obvious energy considerations but also for their higher inherent potential in delivering complex far-field amplitude distributions, annular phase filters are being investigated in different contexts [6-9].

A systematic study on the use of phase filters on annular pupils can be conveniently carried out with the help of annular Walsh filters derived from the annular Walsh functions. Walsh functions form a closed set of normal orthogonal functions over a given finite interval and take on values +1 or -1 , except at a finite number of points of discontinuity, where they take the value zero $[10,11]$. The order of a Walsh function is directly related to the number of its zero crossings or phase transitions within the specified domain, and they constitute a closed set of normal orthogonal functions over the specified interval. They have the interesting property that an approximation of a continuous function over a finite interval by a finite set of Walsh functions leads to a piecewise constant approximation to the function. Walsh filters of various orders may be obtained from corresponding Walsh functions, by realizing transmission values of +1 and -1 by 0 and $\pi$ phase, respectively. Incidentally it may be noted that binary phase filters are being explored for many interesting light distributions [12].

Walsh functions have been used in the field of signal coding and transmission and in allied problems of information processing [13]. Two-dimensional Walsh functions in the usual rectangular coordinates have been used in digital image processing applications [14]. For treatment of problems of optical imaging, Walsh functions in polar co-ordinates have been utilised [15]. For systems with rotational symmetry about the axis, radial Walsh functions [16] have been developed as a special case of Walsh functions in polar co-ordinates and they were proved useful in the treatment of apodization problems $[17,18]$. It has also been observed that not only the transverse amplitude distribution on the far-field plane but also the axial distribution of amplitude/intensity in the farfield is significantly modified in presence of radial Walsh filters on unobscured apertures [19]. 
Annular Walsh functions are a generalization of radial Walsh functions. For a specific central obscuration ratio, they are a complete set of orthogonal functions over the annulus. Annular Walsh filters may be considered to have ternary transmission values. The three values of transmission are zero amplitude over the central obscuration, unity amplitude and zero phase for value +1 , and unity amplitude and $\pi$ phase for value -1 in corresponding annular Walsh functions.

In this paper, we present some results of our investigations on the far-field amplitude characteristics of annular Walsh filters of orders $0,1,2$, and 3 with different central obscuration ratios. After a brief description of annular Walsh functions in the next section, Section 3 presents the mathematical expression for the amplitude distribution in the far-field of an exit pupil with annular Walsh filters on it. Some interesting numerical results and our observations on the same are put forward in the last two sections.

\section{Annular Walsh Functions}

To define annular Walsh function $\Psi_{n}^{\varepsilon}(r)$ of index $n \geq 0$ and argument $r$ over an annular region with $\varepsilon$ and 1 as inner and outer radii, respectively, it is necessary to express the integer $n$ in the form

$$
n=\sum_{m=0}^{\tilde{n}-1} k_{m} 2^{m}
$$

where $k_{m}$ are the bits, 0 or 1 of the binary numeral for $n$, and $\left(2^{\widetilde{n}}\right)$ is the power of 2 that just exceeds $n$. For all $r$ in $(\varepsilon, 1)$, $\Psi_{n}^{\varepsilon}(r)$ is defined as

$$
\Psi_{n}^{\varepsilon}(r)=\prod_{m=0}^{\tilde{n}-1} \operatorname{sgn}\left\{\cos \left[k_{m} 2^{m} \pi \frac{\left(r^{2}-\varepsilon^{2}\right)}{\left(1-\varepsilon^{2}\right)}\right]\right\},
$$

where

$$
\operatorname{sgn}(x)= \begin{cases}+1, & x>0, \\ 0, & x=0, \\ -1, & x<0\end{cases}
$$

The orthogonality condition implies that

$$
\int_{\varepsilon}^{1} \Psi_{m}^{\varepsilon}(r) \Psi_{n}^{\varepsilon}(r) r d r=\frac{1-\varepsilon^{2}}{2} \delta_{m n}
$$

where $\delta_{m n}$ is the Kronecker delta defined as

$$
\delta_{m n}= \begin{cases}0, & m \neq n \\ 1, & m=n\end{cases}
$$

Figure 1 shows the first four annular Walsh functions for central obscuration $\varepsilon=0.3$ in two dimensions. Figure 2 presents values of the functions $\Psi_{n}^{0.3}(r), n=0, \ldots, 3$, along the radius in an azimuthal direction. It should be noted that the order of the functions $n$ is equal to the number of zero crossings, or sign changes of the function in the interval $(0.3,1)$, and

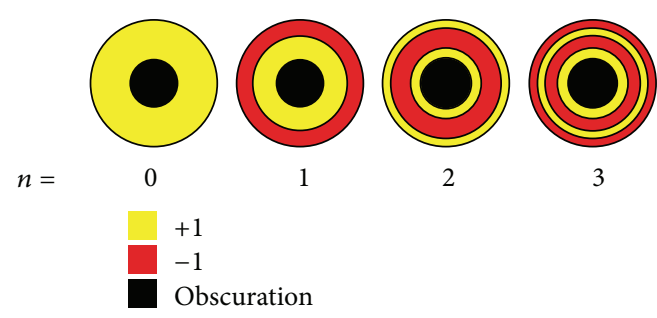

FIGURE 1: Annular Walsh functions $\Psi_{n}^{\varepsilon}(r)$ in two dimensions of order $n=0,1,2,3$ for central obscuration $\varepsilon=0.3$.
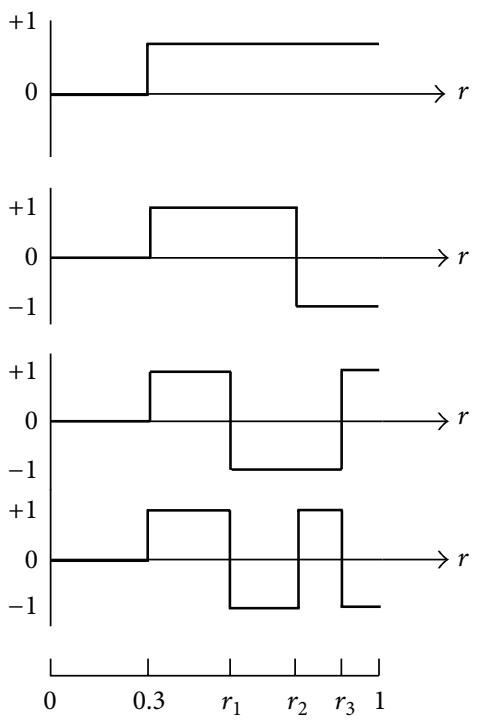

FIGURE 2: Annular Walsh functions $\Psi_{n}^{\varepsilon}(r)$ of order $n=0,1,2,3$ along radius $r$ for central obscuration $\varepsilon=0.3$.

locations of the points of zero crossings for members of the set of functions $\Psi_{n}^{\varepsilon}(r), n=0, \ldots, 3$ are given by

$$
r_{i}=\sqrt{\frac{\left[(4-i) \varepsilon^{2}+i\right]}{4}}, \quad i=1,2,3 .
$$

In general, for the first $N$, where $N=2^{b}$ and $b$ is a positive integer, and annular Walsh functions $\Psi_{n}^{\varepsilon}(r), n=0,1, \ldots,(N-$ $1)$, the zero crossings are located at

$$
r_{i}=\sqrt{\frac{\left[(N-i) \varepsilon^{2}+i\right]}{N}}, \quad i=1,2, \ldots,(N-1) .
$$

The inner and outer radii of the annulus is $r_{0}=\varepsilon$ and $r_{N}=1$. Note that the set of $(N-1)$ zero crossing locations, $r_{i}, i=$ $1,2, \ldots,(N-1)$, consists of all zero crossing locations required for specifying members of this particular set of Walsh functions. An individual member of this set of Walsh functions will have the same number of zero crossings as its order.

For computational purposes it is often convenient to express an annular Walsh function $\Psi_{n}^{\varepsilon}(r)$ as

$$
\Psi_{n}^{\varepsilon}(r)=\sum_{j=1}^{N} h_{n j} \zeta_{j}^{N}(r)
$$


TABLE 1: The Hadamard matrix for $N=2^{2}$.

\begin{tabular}{llll}
\hline+1 & +1 & +1 & +1 \\
+1 & +1 & -1 & -1 \\
+1 & -1 & -1 & +1 \\
+1 & -1 & +1 & -1 \\
\hline
\end{tabular}

where $N=2^{\widetilde{n}}$. For a particular value of $n$, the integer $\widetilde{n}$ is taken such that $N$ just exceeds $n . \zeta_{j}^{N}(r)$ are zero-one functions, also known as Walsh block functions, defined as

$$
\zeta_{j}^{N}(r)= \begin{cases}1, & r_{j-1} \leq r \leq r_{j} \\ 0, & \text { otherwise. }\end{cases}
$$

For all annular Walsh functions of order $n<N$, the same values of $r_{j}, j=0, N$ are to be used. They are unique for the particular value of $N$, and their values are given in (7). $h_{n j}$ are the elements of a $(N \times N)$ Hadamard matrix whose elements are +1 or -1 [13]. Table 1 gives the Hadamard matrix for $N=2^{2}$. The Hadamard matrices for other values of $N$ can be obtained from the defining relation (2) of annular Walsh functions.

\section{Far-Field Amplitude Distribution}

Figure 3 shows the image space of an axially symmetric imaging system. It is well known that the complex amplitude distribution on the image plane corresponding to an axial object point is given by the far-field diffraction pattern of the pupil function over the exit pupil. The complex amplitude $F(p)$ at a point $\mathrm{Q}^{\prime}$ in the far-field due to a point object on the axis is, apart from the multiplicative constant, given by the Hankel transform of order zero of the pupil function [20,21]:

$$
F(p)=\int_{0}^{1} f(r) J_{0}(p r) r d r
$$

where $f(r)$ is the circularly symmetric pupil function. The variable $r$ is the fractional coordinate for a point $P^{\prime}$ on the pupil sphere of center $O^{\prime}$ and radius $O^{\prime} E^{\prime}$, where $E^{\prime}$ is the axial position of the exit pupil. $r$ is equal to the ratio $\left(r^{\prime} / h^{\prime}\right)$, where $r^{\prime}$ is the perpendicular distance of the point $P^{\prime}$ from the axis and $h^{\prime}$ is the maximum value of $r^{\prime}$, so that $0 \leq r \leq 1$. $p$ is the reduced diffraction variable given by

$$
p=\frac{2 \pi}{\lambda}\left(n^{\prime} \sin \alpha^{\prime}\right) \xi^{\prime}
$$

where $\alpha^{\prime}$ is the semiangular aperture of the imaging system, $n^{\prime}$ is the refractive index of the image space, $2 \pi / \lambda$ is the propagation constant, and $\xi^{\prime}\left(=O^{\prime} \mathrm{Q}^{\prime}\right)$ is the actual geometrical distance of point $\mathrm{Q}^{\prime}$ on the image plane from the center $\mathrm{O}^{\prime}$ of the diffraction pattern.

For a specific central obscuration ratio $\varepsilon$, annular Walsh filters of various orders can be obtained from the corresponding annular Walsh functions by realizing transmission values +1 and -1 by zero and $\pi$ phase filters, respectively. In presence

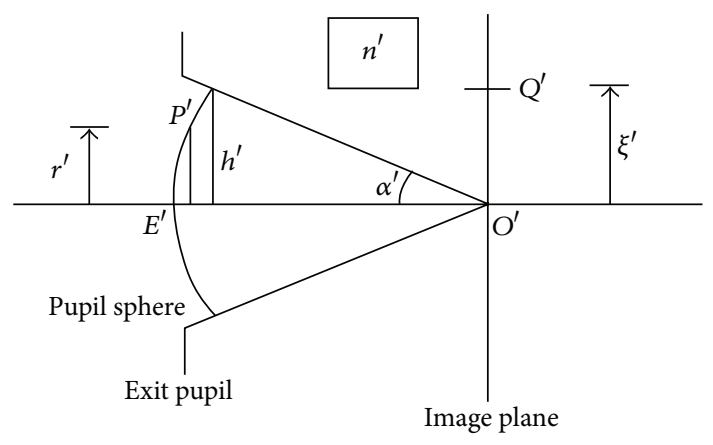

FIGURE 3: Schematic representation of the image space parameters.

of an annular Walsh filter on a uniform pupil, the pupil function $f(r)$ is given by

$$
f(r)= \begin{cases}0, & 0 \leq r<\varepsilon, \\ \Psi_{n}^{\varepsilon}(r) & \varepsilon \leq r<1 .\end{cases}
$$

It may be noted that $f(r)$ is binary (value either 0 or +1 ) only in the case of zero order annular Walsh function $\Psi_{0}^{\varepsilon}(r)$; for all other orders, $f(r)$ is ternary with value either $0,+1$, or -1 .

The far-field amplitude pattern $F_{n}^{\varepsilon}(p)$ of an annular Walsh filter $\Psi_{n}^{\varepsilon}(r)$ is given by

$$
F_{n}^{\varepsilon}(p)=\int_{\varepsilon}^{1} \Psi_{n}^{\varepsilon}(r) J_{0}(p r) r d r=\sum_{j=1}^{N} h_{n j} \Gamma_{j}(p),
$$

where

$$
\begin{aligned}
\Gamma_{j}(p) & =\int_{\varepsilon}^{1} \zeta_{j}^{N}(r) J_{0}(p r) r d r \\
& =\left[\frac{r_{j}^{2} J_{1}\left(p r_{j}\right)}{p r_{j}}-\frac{r_{j-1}^{2}\left(p r_{j-1}\right)}{p r_{j-1}}\right] .
\end{aligned}
$$

\section{Numerical Results and Discussion}

Figures 4-7 give the far-field amplitude distributions for annular Walsh filters $\Psi_{n}^{\varepsilon}(r)$, with central obscuration $\varepsilon=0.3$, $0.5,0.7$, and 0.9 , respectively. In each figure, the amplitude distribution is shown for the annular Walsh filters of orders 0 , 1,2 , and 3 . Note that the amplitude values are normalized by the central amplitude for an unobstructed uniform aperture. For the sake of underscoring amplitude variations, the scales along the ordinate and abscissa of different curves could not be maintained the same; they were tailored to bring forth the specific features.

It is seen in Figures 4(a), 5(a), 6(a), and 7(a) that for the zero order annular Walsh filters, there is a central maximum of amplitude in the diffraction pattern, and the magnitude of the maximum is obviously dependent on the central obscuration $\varepsilon$. For the sake of comparison, amplitude distribution for an unobscured uniform aperture is shown alongside the same for zero order annular Walsh filters. It is well known that with higher values of obscuration, the central lobe narrows down significantly [20]. 


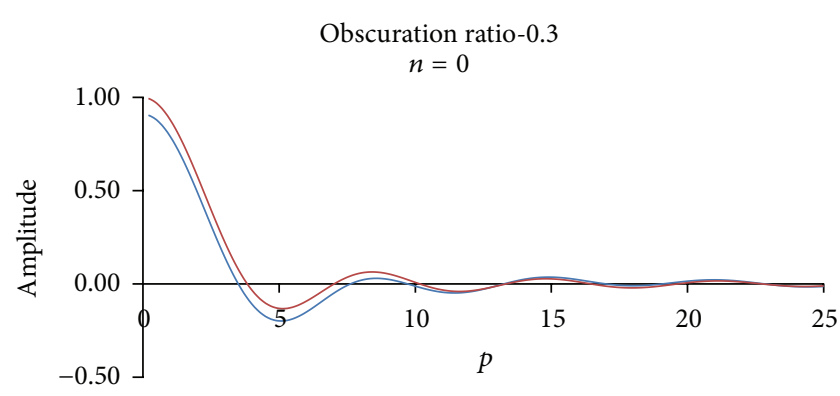

(a)

Obscuration ratio- 0.3
$n=2$

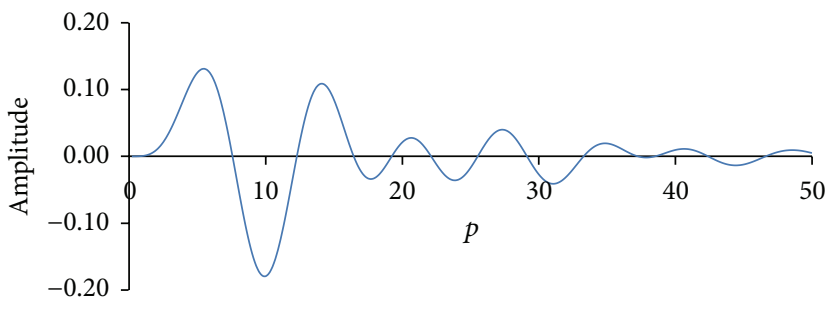

(c)

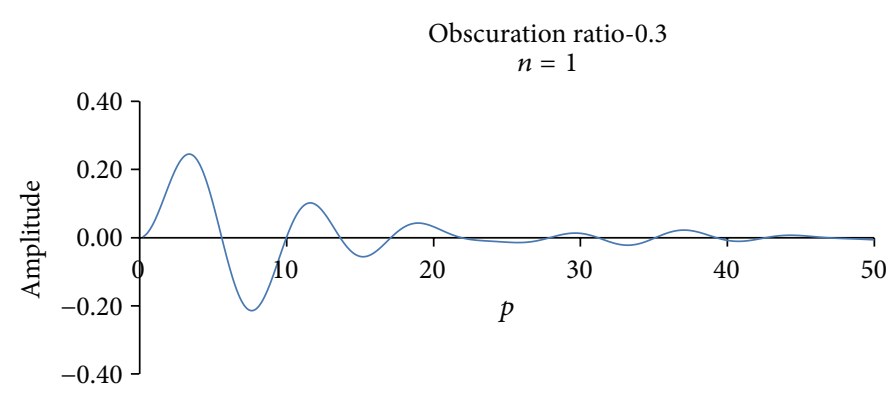

(b)

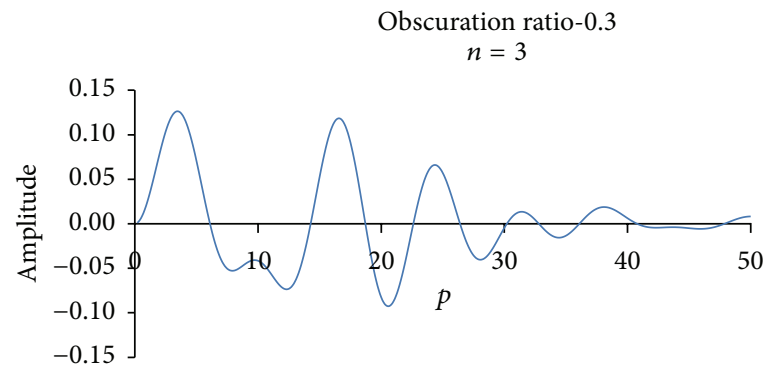

(d)

FIGURE 4: Far-field amplitude distributions for annular Walsh filters $\Psi_{n}^{\varepsilon}(r)$ on an annular aperture with central obscuration $\varepsilon=0.3$ for orders (a) $n=0$, (b) $n=1$, (c) $n=2$, and (d) $n=3$. The red graph in (a) represents amplitude distribution for an unobscured uniform aperture.

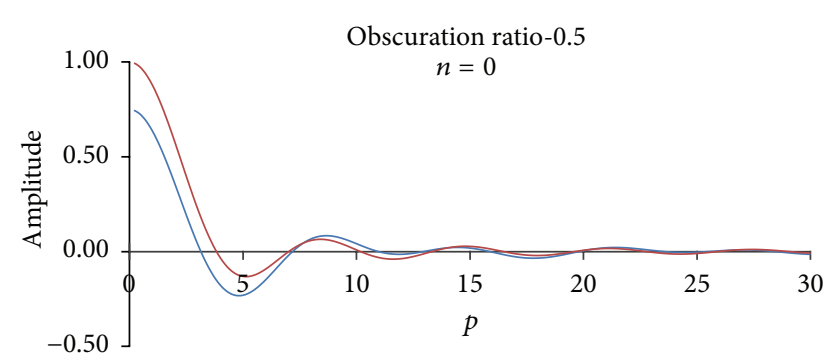

(a)

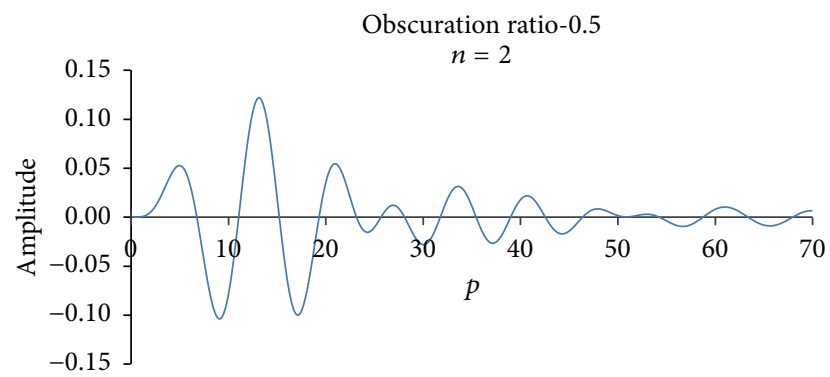

(c)

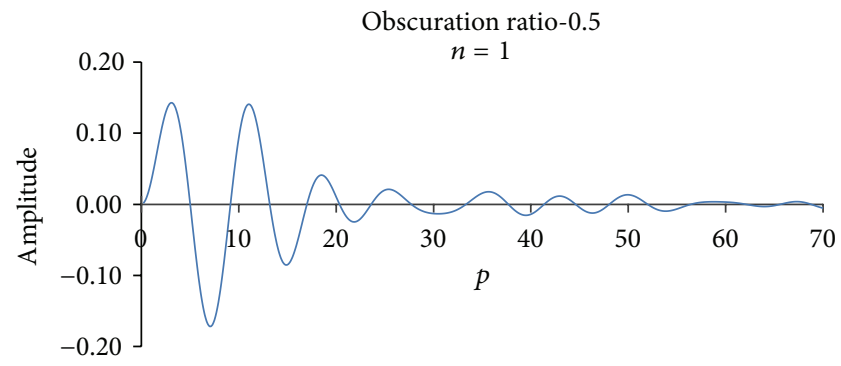

(b)

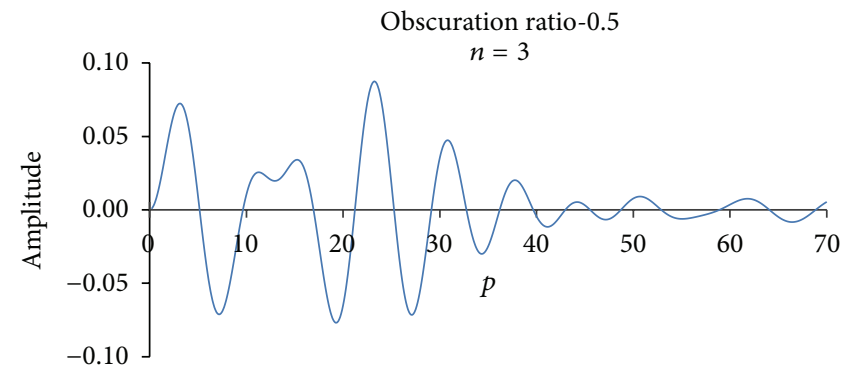

(d)

FIGURE 5: Far-field amplitude distributions for annular Walsh filters $\Psi_{n}^{\varepsilon}(r)$ on an annular aperture with central obscuration $\varepsilon=0.5$ for orders (a) $n=0$, (b) $n=1$, (c) $n=2$, and (d) $n=3$. The red graph in (a) represents amplitude distribution for an unobscured uniform aperture.

Figures 4-7 show that for Walsh filters of all orders other than zero, the central amplitude is zero. This is a consequence of orthogonality property of the Walsh functions. The dark center is surrounded by rings of oscillating amplitudes. For lower orders, this oscillation gradually decays after the first few rings, but for higher orders, the oscillations continue for many more rings. As expected from energy considerations, the amplitude of oscillation is significantly less in case of higher order filters compared to the lower order ones in each case of obscuration. From lower to higher obscuration, the ring with peak oscillatory amplitude gradually shifts away from the centre in case of higher order annular Walsh filters. 


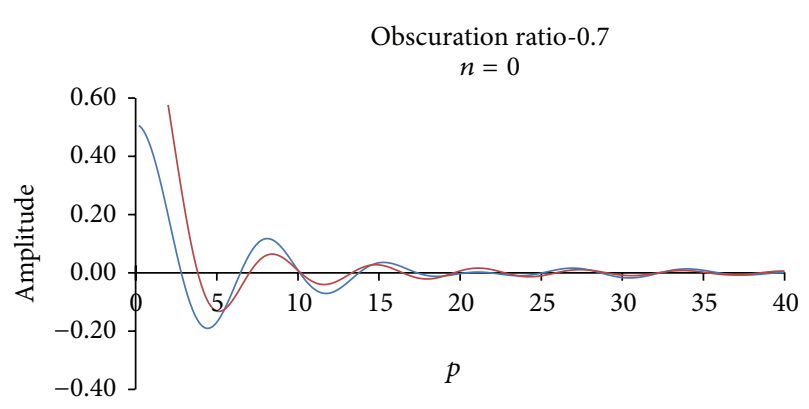

(a)

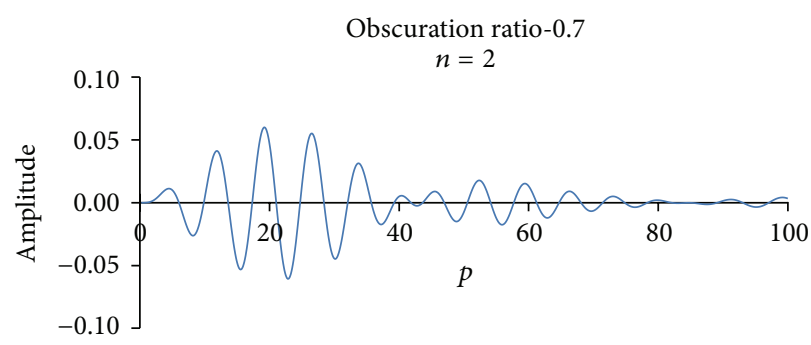

(c)

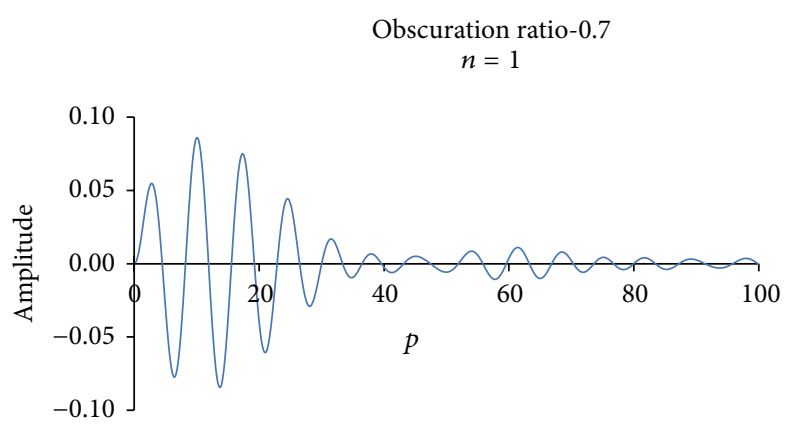

(b)

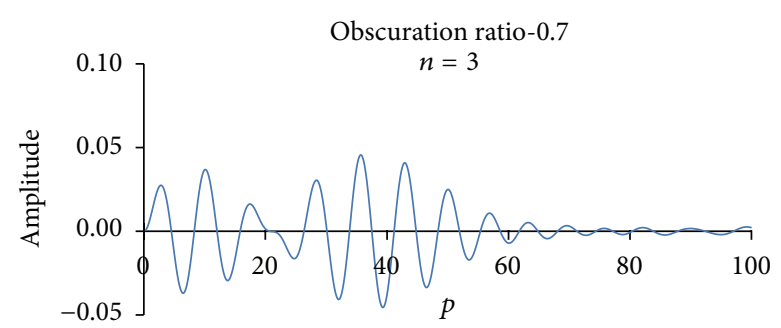

(d)

FiguRE 6: Far-field amplitude distributions for annular Walsh filters $\Psi_{n}^{\varepsilon}(r)$ on an annular aperture with central obscuration $\varepsilon=0.7$ for orders (a) $n=0$, (b) $n=1$, (c) $n=2$, and (d) $n=3$. The red graph in (a) represents amplitude distribution for an unobscured uniform aperture.

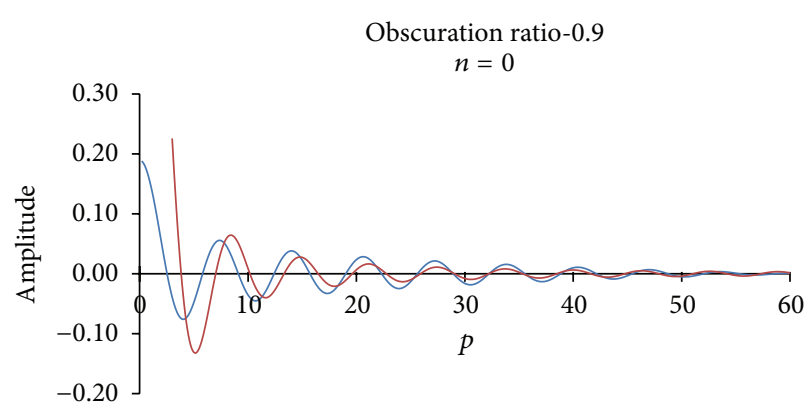

(a)

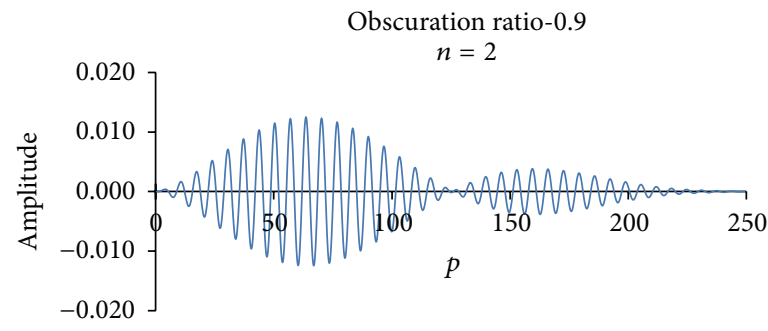

(c)

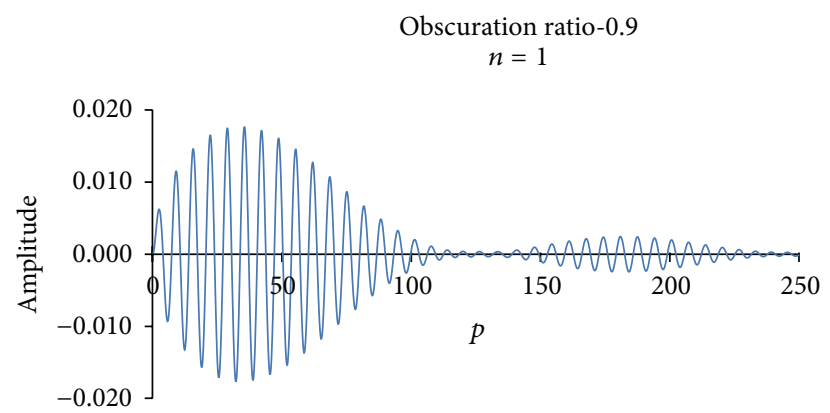

(b)

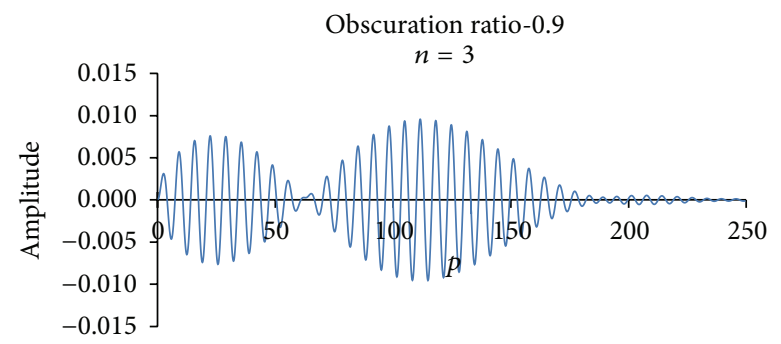

(d)

FIGURE 7: Far-field amplitude distributions for annular Walsh filters $\Psi_{n}^{\varepsilon}(r)$ on an annular aperture with central obscuration $\varepsilon=0.9$ for orders (a) $n=0$, (b) $n=1$, (c) $n=2$, and (d) $n=3$. The red graph in (a) represents amplitude distribution for an unobscured uniform aperture.

Use of ternary values of transmission $0,+1$, and -1 in annular Walsh filters has opened up new possibilities for generation of interesting far-field patterns. Amplitude patterns shown in Figures 6(b)-6(d) and 7(b)-7(d) corresponding to annular Walsh filters of orders 1, 2, and 3 on a pupil with large obscuration have distinct characteristics that remain to be explored further for many practical applications, for example, optical encryption. Incidentally, it is also observed that for orders higher than 3 , the typical nature of amplitude distribution is more pronounced, with the frequency of oscillations in amplitude becoming increasingly higher with concomitant decrease in peak amplitude. 


\section{Concluding Remarks}

Optimal combinations of annular Walsh filters can help in realizing desired amplitude distributions on the far-field plane for applications using coherent illumination. An important application is to obtain "superresolution" effect by narrowing down of the central lobe and suppression of the side ripples. Solution of the related optimization problem can make effective use of orthogonality of annular Walsh functions. Incidentally, inverse problems on pupil synthesis for prespecified intensity characteristics in the far-field plane were tackled earlier for the case of incoherent illumination by the use of radial Walsh filters for unobscured apertures [18]. It is obvious that the availability of an additional degree of freedom, namely, the obscuration ratio $\varepsilon$, is likely to expand the scope of this inverse problem.

Ready availability of high efficiency spatial light modulators has facilitated the practical realization of phase filters. [22-24]. Nevertheless, it is obvious that, in general, practical implementation of filters with binary or ternary phase values is relatively easier than filters with continuously varying phase. Use of Walsh function-based analysis and synthesis of pupil plane filters has become useful, for it can circumvent the tricky problem of synthesizing continuously varying phase by providing alternative analytical treatments that deal with a finite number of discrete phase levels.

Finally, it remains to investigate the axial resolution characteristics of annular Walsh filters and also their usefulness in the synthesis of three-dimensional light fields [25].

\section{Acknowledgment}

Authors are indebted to the anonymous reviewers whose comments have significantly improved the quality of our presentation.

\section{References}

[1] G. T. di Francia, "Nuovo pupille superrisolvente," Atti della Fondazione Giorgio Ronchi, vol. 7, pp. 366-372, 1952.

[2] E. H. Linfoot and E. Wolf, "Diffraction images in systems with an annular aperture," Proceedings of the Physical Society B, vol. 66, no. 2, pp. 145-149, 1953.

[3] A. Boivin, Théorie et Calcul des Figures de Diffraction de Revolution, Gauthier-Villars, Paris, France, 1964.

[4] Z. S. Hegedus, "Annular pupil arrays-application to confocal scanning," Optica Acta, vol. 32, pp. 815-826, 1985.

[5] C. J. R. Sheppard and A. Choudhury, "Annular pupils, radial polarization, and superresolution," Applied Optics, vol. 43, no. 22, pp. 4322-4327, 2004.

[6] T. R. M. Sales and G. M. Morris, "Diffractive superresolution elements," Journal of the Optical Society of America A, vol. 14, no. 7, pp. 1637-1646, 1997.

[7] H. Luo and C. Zhou, "Comparison of superresolution effects with annular phase and amplitude filters," Applied Optics, vol. 43, no. 34, pp. 6242-6247, 2004.

[8] M. Yun, L. Liu, J. Sun, and D. Liu, "Three-dimensional superresolution by three-zone complex pupil filters," Journal of the Optical Society of America A, vol. 22, no. 2, pp. 272-277, 2005.
[9] M. Yun, M. Wang, and L. Liu, "Super-resolution with annular binary phase filter in the 4Pi-confocal system," Journal of Optics A, vol. 7, no. 11, pp. 640-644, 2005.

[10] J. L. Walsh, "A closed set of normal orthogonal functions," The American Journal of Mathematics, vol. 55, pp. 5-24, 1923.

[11] K. G. Beauchamp, Walsh Functions and Their Applications, Academic, New York, NY, USA, 1985.

[12] C. J. R. Sheppard, "Binary phase filters with a maximally-flat response," Optics Letters, vol. 36, no. 8, pp. 1386-1388, 2011.

[13] H. F. Harmuth, Transmission of Information by Orthogonal Functions, Springer, Berlin, Germany, 1972.

[14] H. C. Andrews, Computer Techniques in Image Processing, Academic, New York, NY, USA, 1970.

[15] M. De and L. N. Hazra, "Walsh functions in problems of optical imagery," Optica Acta, vol. 24, pp. 221-234, 1977.

[16] L. N. Hazra and A. Guha, "Farfield diffraction properties of radial Walsh filters," Journal of the Optical Society of America A, vol. 3, pp. 843-846, 1986.

[17] L. N. Hazra and A. Banerjee, "Application of Walsh function in generation of optimum apodizers," Journal of Optics, vol. 5, pp. 19-26, 1976.

[18] L. N. Hazra, "A new class of optimum amplitude filters," Optics Communications, vol. 21, no. 2, pp. 232-236, 1977.

[19] L. N. Hazra, "Walsh filters for tailoring of resolution in microscopic imaging," Micron, vol. 38, no. 2, pp. 129-135, 2007.

[20] M. Born and E. Wolf, Principles of Optics, Pergamon, Oxford, UK, 1980.

[21] H. H. Hopkins, "Canonical and real space coordinates used in the theory of image formation," in Applied Optics and Optical Engineering, R. R. Shannon and J. C. Wyant, Eds., vol. 9, p. 307, Academic, New York, NY, USA, 1983.

[22] E. Fernández, A. Márquez, S. Gallego, R. Fuentes, C. García, and I. Pascua, "Hybrid ternary modulation applied to multiplexing holograms in photopolymers for data page storage," Journal of Lightwave Technology, vol. 28, pp. 776-783, 2010.

[23] A. Au, C.-S. Wu, S.-T. Wu, and U. Efron, "Ternary phase and amplitude modulations using a twisted nematic liquid crystal spatial light modulator," Applied Optics, vol. 34, no. 2, pp. 281284, 1995.

[24] S. Mukhopadhyay, S. Sarkar, K. Bhattacharya, and L. N. Hazra, "Polarisation phase shifting interferometric technique for phase calibration of a reflective phase spatial light modulator," Optical Engineering, vol. 52, Article ID 035602, 2 pages, 2013.

[25] R. Piestun and J. Shamir, "Synthesis of three-dimensional light fields and applications," Proceedings of the IEEE, vol. 90, no. 2, pp. $222-244,2002$. 

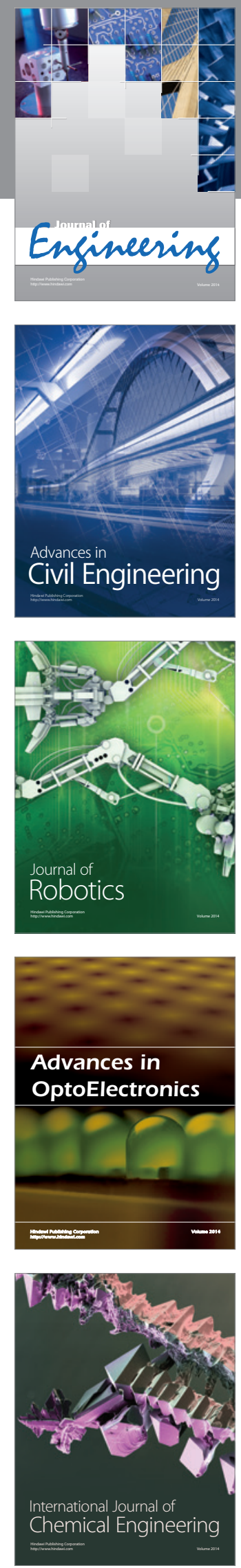

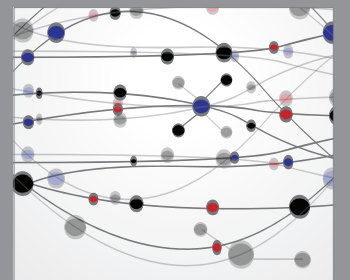

The Scientific World Journal
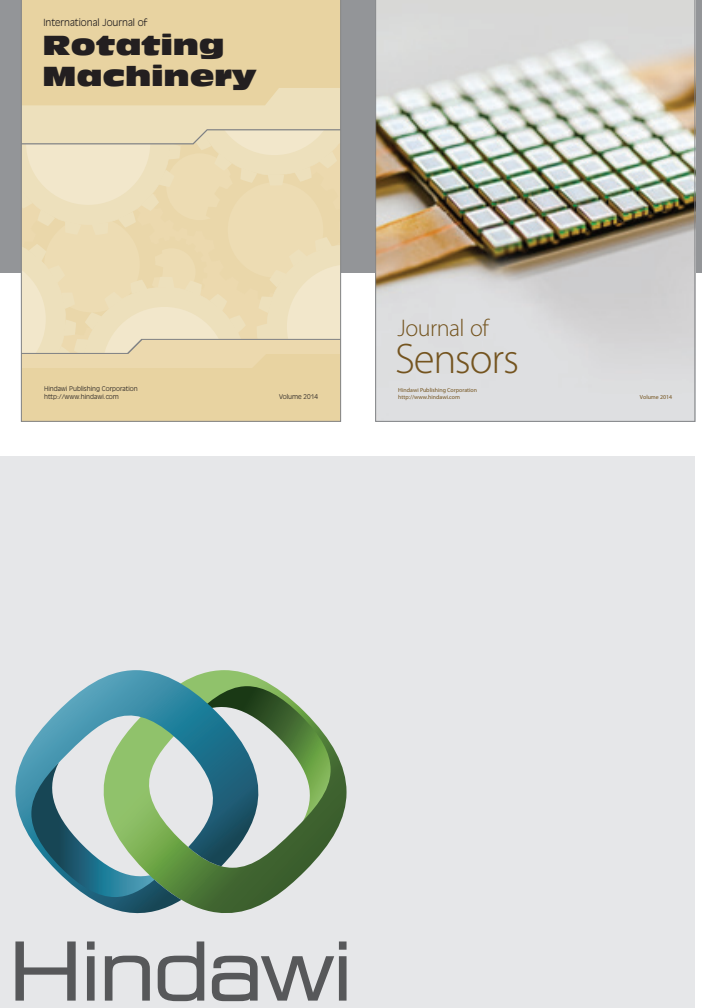

Submit your manuscripts at http://www.hindawi.com
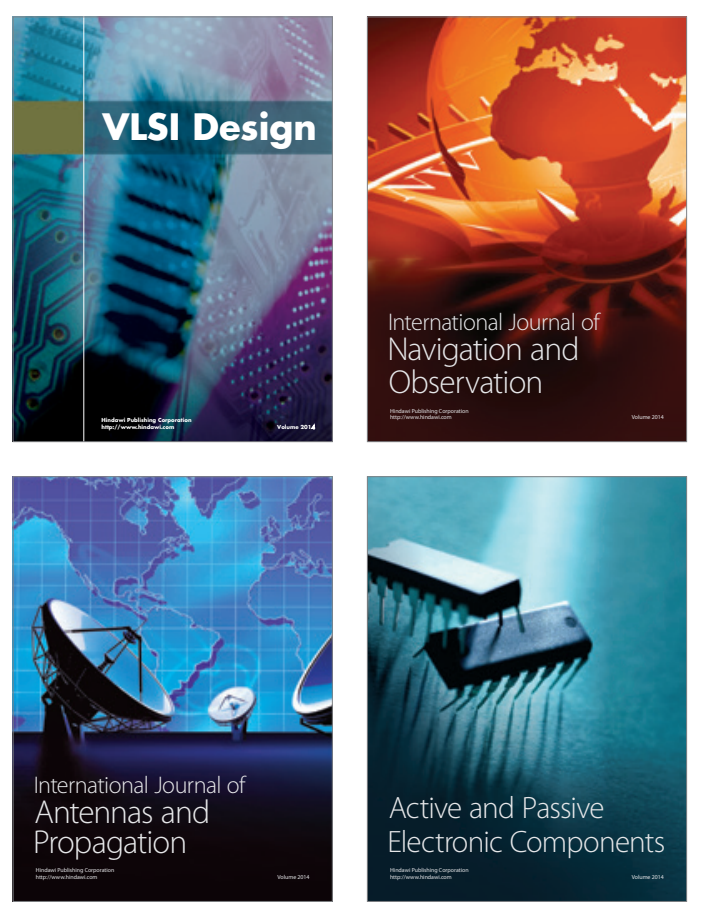
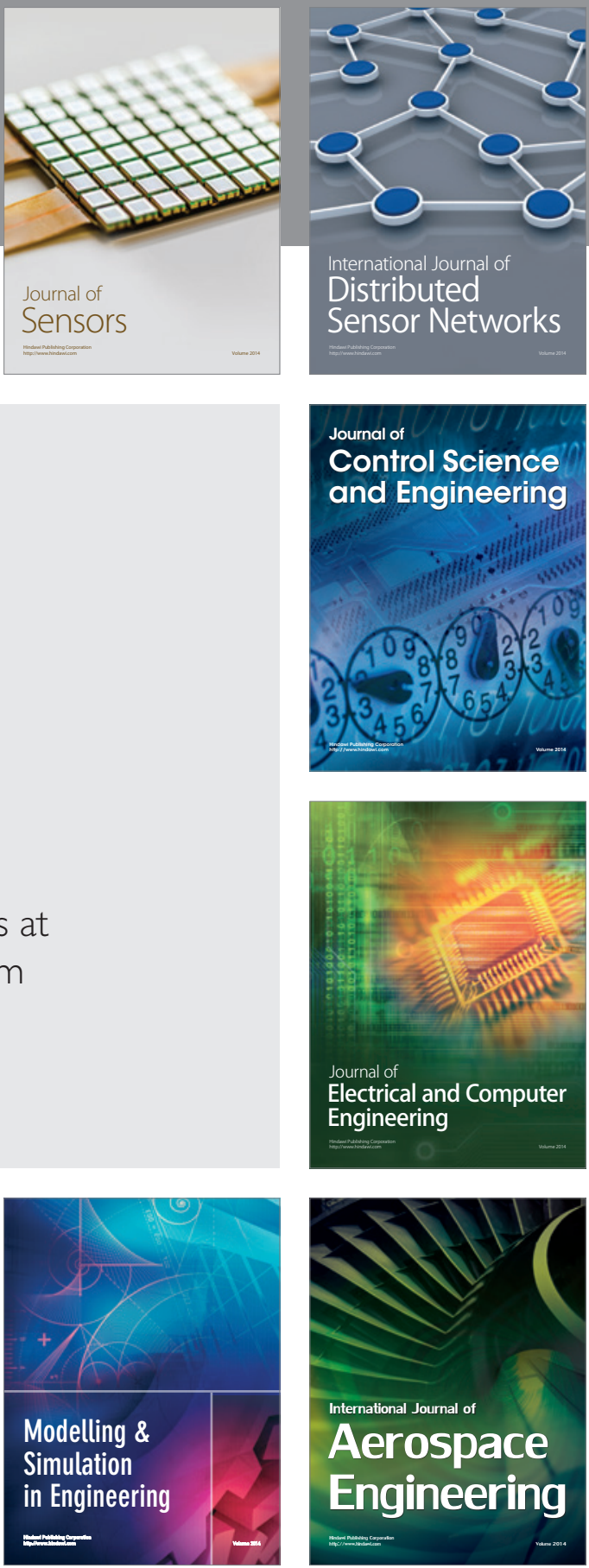

Journal of

Control Science

and Engineering
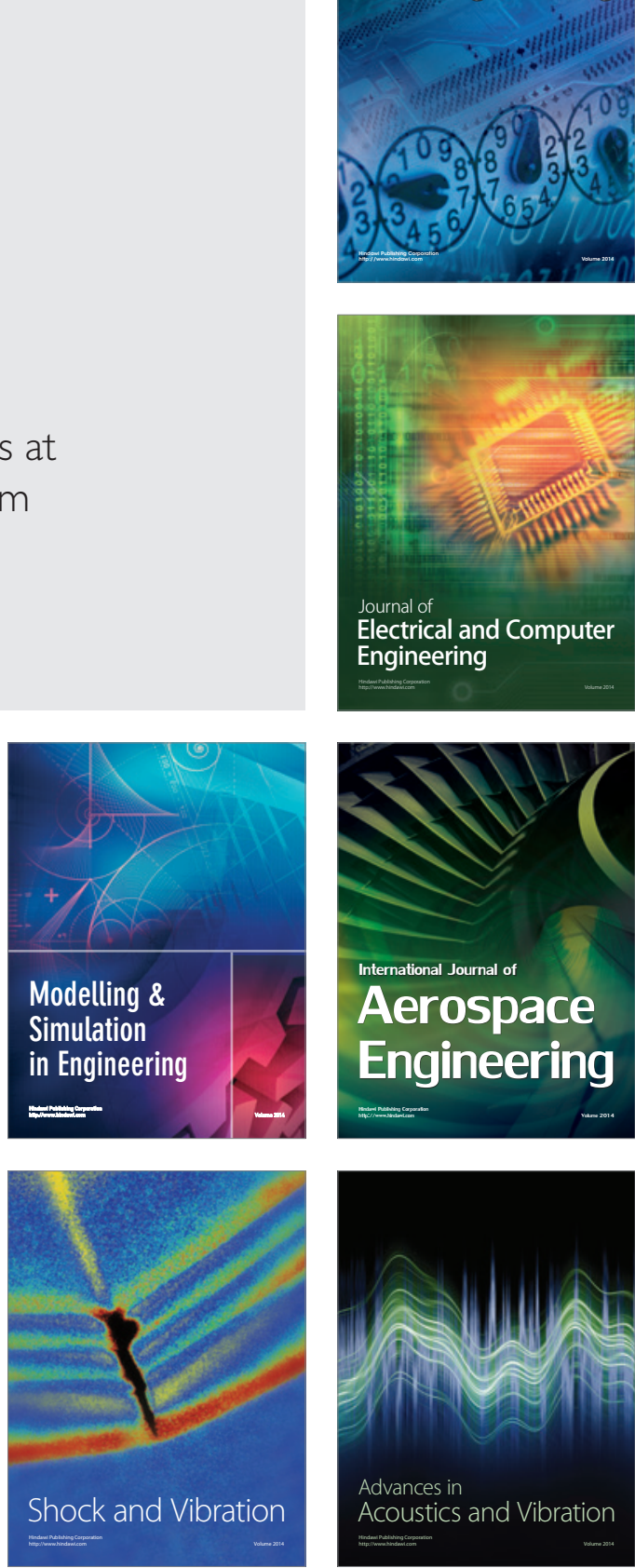